PROCEEDINGS OF THE

AMERICAN MATHEMATICAL SOCIETY

Volume 137, Number 7, July 2009, Pages 2387-2392

S 0002-9939(08)09779-7

Article electronically published on December 23, 2008

\title{
ASYMPTOTIC BEHAVIOR OF NONEXPANSIVE MAPPINGS IN FINITE DIMENSIONAL NORMED SPACES
}

\author{
BRIAN LINS
}

(Communicated by Marius Junge)

\begin{abstract}
If $X$ is a finite dimensional real normed space, $C$ is a closed convex subset of $X$ and $f: C \rightarrow C$ is nonexpansive with respect to the norm on $X$, then we show that either $f$ has a fixed point in $C$ or there is a linear functional $\varphi \in X^{*}$ such that $\lim _{k \rightarrow \infty} \varphi\left(f^{k}(x)\right)=\infty$ for all $x \in C$.
\end{abstract}

\section{INTRODUCTION}

Let $X$ be a real normed space with norm $\|\cdot\|$. Let $X^{*}$ denote the dual space of $X$. Suppose that $U$ is a subset of $X$. We say that a map $f: U \rightarrow X$ is nonexpansive if $\|f(x)-f(y)\| \leq\|x-y\|$ for all $x, y \in U$.

For a nonexpansive map $f: U \rightarrow U$, if $\chi=\lim _{k \rightarrow \infty} f^{k}(x) / k$ exists for some $x \in U$, then $\lim _{k \rightarrow \infty} f^{k}(y) / k=\chi$ for all $y \in U$. The limit $\chi$ is called the cycle time vector. For examples where the cycle time vector does not exist, see the proof of Theorem 1.4 in [10, section 4 in [15], and also [6]. Even if the cycle time vector fails to exist, it is known (Lemma 1, 13]) that there is a constant $A=\lim _{k \rightarrow \infty}\left\|f^{k}(x) / k\right\|$, and $A$ does not depend on $x$. We call this constant the linear rate of growth of $f$.

The following theorem is due to Kohlberg and Neyman (Theorem 1.1 in [10]).

Theorem 1.1 (Kohlberg and Neyman 10]). Let $C$ be a convex subset of a normed space $X$ and let $f: C \rightarrow C$ be nonexpansive. Then there exists a linear functional $\varphi \in X^{*}$ with $\|\varphi\|=1$ such that for every $x \in C$,

$$
\lim _{k \rightarrow \infty} \varphi\left(\frac{f^{k}(x)}{k}\right)=\lim _{k \rightarrow \infty}\left\|\frac{f^{k}(x)}{k}\right\|=\inf _{y \in C}\|f(y)-y\| .
$$

If the linear rate of growth $A=\lim _{k \rightarrow \infty}\left\|f^{k}(x) / k\right\|>0$, then there is a linear functional $\varphi \in X^{*}$ such that $\lim _{k \rightarrow \infty} \varphi\left(f^{k}(x)\right)=\infty$. Even more can be said if $X$ is a reflexive Banach space. In that case, Rouhani and Kirk have shown (Theorem 1, [18]) that if $U \subset X$ and $f: U \rightarrow U$ is nonexpansive, then the weak topology accumulation points of the sequence $f^{k}(x) / k, k \geq 1$ are contained in a convex subset of the sphere centered at the origin with radius equal to the linear rate of growth of $f$. Related results can be found in [10, as well as Proposition 1 in [13] and the paragraph before Proposition 4.4 in [16].

Received by the editors July 23, 2007, and, in revised form, September 28, 2008.

2000 Mathematics Subject Classification. Primary 47H09.

(C)2008 American Mathematical Society Reverts to public domain 28 years from publication 
If the linear rate of growth of $f$ is zero, these theorems tell us relatively little about the asymptotic behavior of $f$. Of course, if $f$ has a fixed point, then the linear rate of growth $A=0$. Certain special classes of maps, such as piecewise affine maps (Theorem 2.1, 9]), have a fixed point whenever $A=0$. However, there are simple examples of nonexpansive maps with $A=0$ which have no fixed points. For example, the map

$$
f(x)=\left\{\begin{array}{cc}
x+1 / x & x>1 \\
x+1 & x \leq 1
\end{array}\right.
$$

is a nonexpansive map on $\mathbb{R}$ with no fixed points, even though the linear rate of growth of $f$ is zero.

The main result of this paper is the following theorem.

Theorem 1.2. Suppose that $C$ is a closed convex set in a finite dimensional real normed space $X$ and $f: C \rightarrow C$ is a nonexpansive map. If $f$ does not have a fixed point in $C$, then there is a linear functional $\varphi \in X^{*}$ such that $\lim _{k \rightarrow \infty} \varphi\left(f^{k}(x)\right)=\infty$ for all $x \in C$.

Note that Theorem 1.2 is closely related to Theorem 1.1 although Theorem 1.2 only applies to finite dimensional normed spaces. For infinite dimensional counterexamples to Theorem 1.2, see Theorems 2.1 and 2.2 in [5]. The advantage of Theorem 1.2 is that it tells us something about the asymptotic behavior of fixed point free nonexpansive maps, even when the linear rate of growth is zero.

\section{THE HOROFUNCTION BOUNDARY}

The main tool we will use in this paper is the horofunction boundary of $X$. The horofunction boundary has been used to study the asymptotic behavior of unbounded nonexpansive maps in several settings (see [1], 7], 11, 12]).

We will define the horofunction boundary for a finite dimensional real normed space $X$. Let $C(X)$ denote the set of continuous real-valued maps on $X$ endowed with the topology of uniform convergence on compacta. Define $\Phi$ to be the map $\Phi: x \mapsto g_{x}$, where

$$
g_{x}(y)=\|y-x\|-\|x\| \text {. }
$$

Note that $\Phi$ is a continuous embedding of $X$ into $C(X)$. Since $X$ is not compact, the image $\Phi(X)$ will not be closed in $C(X)$. The closure of $\Phi(X)$ is called the Busemann compactification of $X$, and we denote it by $\operatorname{cl} \Phi(X)$. The boundary of $X$ under this compactification is $X(\infty)=\operatorname{cl} \Phi(X) \backslash \Phi(X)$. The elements of $X(\infty)$ are called horofunctions. Note that any horofunction $h \in X(\infty)$ can be written

$$
h(y)=\lim _{k \rightarrow \infty}\left\|y-x_{k}\right\|-\left\|x_{k}\right\|,
$$

where $\left\langle x_{k}\right\rangle_{k>0}$ is a sequence of points in $X$ such that the limit above converges uniformly to $h$ on compact sets. For more details about this compactification, see 2]; and for more information about the horofunction boundary of finite dimensional normed spaces, see [8] and 19 .

In order to prove Theorem 1.2 we must first establish the following relationship between nonexpansive maps and the horofunctions on $X$.

Theorem 2.1. Let $C$ be a closed convex subset of a finite dimensional real normed space $X$. Let $f: C \rightarrow C$ be nonexpansive and suppose that $f$ has no fixed point in $C$. Then there is a horofunction $h \in X(\infty)$ such that $\lim _{k \rightarrow \infty} h\left(f^{k}(x)\right)=-\infty$ for all $x \in C$. 
The proof of Theorem 2.1 depends on the following two lemmas. Note that Lemma 2.1 is a special case of Theorem 1 in [14].

Lemma 2.1. Suppose that $C$ is a closed convex subset of $X$ and $f: C \rightarrow C$ is nonexpansive. If $f$ has no fixed point in $C$, then for every $x \in C$ the sequence $\left\langle f^{k}(x)\right\rangle_{k \geq 0}$ is not bounded.

Proof. Suppose that there is a constant $M>0$ such that $\left\|f^{k}(x)\right\|<M$ for all $k \geq 0$. Let $\omega$ denote the set of accumulation points of the sequence $\left\langle f^{k}(x)\right\rangle_{k \geq 0}$. Note that $\omega$ is nonempty, closed and bounded. Dafermos and Slemrod have shown (Theorem 1, 4]) that $f$ is an invertible isometry on $\omega$ and $f$ maps $\omega$ onto itself. For each $y \in \omega$ and $R>0$, let $B_{R}(y)=\{z \in C:\|y-z\| \leq R\}$. Choose $R>2 M$ and let $U=\bigcap_{y \in \omega} B_{R}(y)$. Then $U$ is a nonempty, compact, convex subset of $X$. Since $f$ is nonexpansive and $f(\omega)=\omega$, it follows that $f(U) \subset U$. Therefore, the Brouwer fixed point theorem implies that $f$ has a fixed point in $U$, which is a contradiction.

Remark 2.1. Całka has shown (Theorem 5.6, 3 ) that if $f$ is a nonexpansive map on a finitely totally bounded metric space and $\left\langle f^{k}(x)\right\rangle_{k \geq 0}$ contains a bounded subsequence, then $\left\langle f^{k}(x)\right\rangle_{k \geq 0}$ is bounded. In particular, this result applies to finite dimensional normed spaces and therefore the conclusion of Lemma 2.1 can be strengthened to state that $\lim _{k \rightarrow \infty}\left\|f^{k}(x)\right\|=\infty$.

Lemma 2.2. Let $y \in X$ be an element with $\|y\|=1$. Let $0<\lambda<1$. For any $R>r>0$ and any $z \in X$ with $\|z\| \leq R$, if $\|z-R y\| \leq \lambda R$, then $\|z-r y\| \leq$ $R-(1-\lambda) r$.

Proof. Suppose that $\|z-r y\|>R-(1-\lambda) r$. By the Hahn-Banach theorem there is some $\varphi \in X^{*}$ with $\|\varphi\|=1$ and $\|z-r y\|=\varphi(z-r y)>R-(1-\lambda) r$. Then, $\varphi(z)-\varphi(r y)>R-(1-\lambda) r$, so $\varphi(r y)<\varphi(z)-R+(1-\lambda) r$. Since $\varphi(z) \leq\|z\| \leq R$ it follows that $\varphi(r y)<(1-\lambda) r$ and hence $\varphi(y)<(1-\lambda)$. By scaling, $(R-r) \varphi(y)=\varphi(R y-r y)<(1-\lambda)(R-r)$. So

$$
\varphi(z-R y)=\varphi(z-r y)-\varphi(R y-r y)>R-(1-\lambda) r-(1-\lambda)(R-r)=\lambda R .
$$

Since $\|z-R y\| \geq \varphi(z-R y)>\lambda R$, we have a contradiction.

Proof of Theorem 2.1. Fix an $x_{0} \in C$ and let $x_{k}=f^{k}\left(x_{0}\right)$ for $k \geq 1$. By Lemma2.1 the sequence $\left\langle x_{k}\right\rangle_{k \geq 0}$ is not bounded. Therefore, we may choose an increasing sequence of integers $\left\langle k_{i}\right\rangle_{i \geq 1}$ such that $\lim _{i \rightarrow \infty}\left\|x_{k_{i}}\right\|=\infty$ and, for each $i \geq 1$,

$$
\left\|x_{k_{i}}\right\|>\left\|x_{m}\right\| \text { for all } m<k_{i} \text {. }
$$

Furthermore, since the unit sphere in $X$ is compact, we may also choose $\left\langle k_{i}\right\rangle$ so that there is a $\bar{y} \in X,\|\bar{y}\|=1$, such that

$$
\left\|\frac{x_{k_{i}}}{\left\|x_{k_{i}}\right\|}-\bar{y}\right\| \leq 2^{-i} \text { for all } i \geq 1 \text {. }
$$

Given such a sequence $\left\langle k_{i}\right\rangle$, we claim that

$$
\left\|x_{k_{i}}-x_{k_{j}-m}\right\| \leq\left\|x_{k_{j}}\right\|-\frac{1}{4}\left\|x_{k_{i}}\right\|
$$

whenever $i$ and $m$ are fixed, $m \geq 0$ and $j$ is sufficiently large. Assume this claim for now. 
Since $X$ is locally compact, the Arzelà-Ascoli theorem implies that by taking a refinement of $\left\langle k_{i}\right\rangle$ we may assume the horofunction $h$ defined below exists for all $x \in X:$

$$
h(x)=\lim _{j \rightarrow \infty}\left\|x-x_{k_{j}}\right\|-\left\|x_{k_{j}}\right\| .
$$

By the nonexpansiveness of $f$, we observe that

$$
h\left(x_{k_{i}+m}\right)=\lim _{j \rightarrow \infty}\left\|x_{k_{i}+m}-x_{k_{j}}\right\|-\left\|x_{k_{j}}\right\| \leq \liminf _{j \rightarrow \infty}\left\|x_{k_{i}}-x_{k_{j}-m}\right\|-\left\|x_{k_{j}}\right\| .
$$

Now, by eq. (2.4), $\left\|x_{k_{i}}-x_{k_{j}-m}\right\| \leq\left\|x_{k_{j}}\right\|-\frac{1}{4}\left\|x_{k_{i}}\right\|$ for $j$ sufficiently large. Therefore we obtain

$$
h\left(x_{k_{i}+m}\right) \leq-\frac{1}{4}\left\|x_{k_{i}}\right\| .
$$

This implies that $\lim _{m \rightarrow \infty} h\left(x_{m}\right)=\lim _{m \rightarrow \infty} h\left(f^{m}\left(x_{0}\right)\right)=-\infty$.

Now suppose that $x$ is any element in $C$. Then

$$
\begin{gathered}
h\left(f^{m}(x)\right)=\lim _{j \rightarrow \infty}\left\|f^{m}(x)-x_{k_{j}}\right\|-\left\|x_{k_{j}}\right\| \\
\leq \liminf _{j \rightarrow \infty}\left\|f^{m}(x)-f^{m}\left(x_{0}\right)\right\|+\left\|f^{m}\left(x_{0}\right)-x_{k_{j}}\right\|-\left\|x_{k_{j}}\right\| \\
\leq\left\|f^{m}(x)-f^{m}\left(x_{0}\right)\right\|+h\left(f^{m}\left(x_{0}\right)\right) \leq\left\|x-x_{0}\right\|+h\left(f^{m}\left(x_{0}\right)\right)
\end{gathered}
$$

by the nonexpansiveness of $f$. In particular, $\lim _{m \rightarrow \infty} h\left(f^{m}(x)\right)=-\infty$ since $\lim _{m \rightarrow \infty} h\left(f^{m}\left(x_{0}\right)\right)=-\infty$.

It remains to prove eq. (2.4). For each $i \geq 1$, let $R_{i}=\left\|x_{k_{i}}\right\|$. With this notation, we can rewrite eq. (2.3) as

$$
\left\|x_{k_{i}}-R_{i} \bar{y}\right\| \leq 2^{-i}|| x_{k_{i}} \|, \forall i \geq 1 .
$$

Fix some $i \geq 1$ and $m \geq 0$. Note that

$$
\begin{gathered}
\left\|x_{k_{j}-m}-R_{j} \bar{y}\right\| \leq\left\|x_{k_{j}-m}-x_{k_{j}}\right\|+\left\|x_{k_{j}}-R_{j} \bar{y}\right\| \\
\leq\left\|x_{k_{j}-m}-x_{k_{j}}\right\|+2^{-j}\left\|x_{k_{j}}\right\| \leq \sum_{l=1}^{m}\left\|x_{k_{j}-m+l-1}-x_{k_{j}-m+l}\right\|+2^{-j}\left\|x_{k_{j}}\right\| \\
\leq \sum_{l=1}^{m}\left\|f^{l-1}(x)-f^{l}(x)\right\|+2^{-j}\left\|x_{k_{j}}\right\| \leq m\|f(x)-x\|+2^{-j}\left\|x_{k_{j}}\right\|
\end{gathered}
$$

by the nonexpansiveness of $f$ and the triangle inequality. Thus

$$
\left\|x_{k_{j}-m}-R_{j} \bar{y}\right\| \leq m\|f(x)-x\|+2^{-j}\left\|x_{k_{j}}\right\| .
$$

Since $m\|f(x)-x\|$ is a constant and $\lim _{j \rightarrow \infty}\left\|x_{k_{j}}\right\|=\infty$, equation (2.6) implies that for $j$ large enough, $\left\|x_{k_{j}-m}-R_{j} \bar{y}\right\| \leq \frac{1}{4}\left\|x_{k_{j}}\right\|$. Furthermore, $\left\|x_{k_{j}-m}\right\| \leq\left\|x_{k_{j}}\right\|$ by eq. (2.2). Using Lemma 2.2 with $\lambda=\frac{1}{4}, r=\left\|x_{k_{i}}\right\|, R=\left\|x_{k_{j}}\right\|, y=\bar{y}$ and $z=x_{k_{j}-m}$ we obtain

$$
\left\|x_{k_{j}-m}-R_{i} \bar{y}\right\| \leq\left\|x_{k_{j}}\right\|-\frac{3}{4}\left\|x_{k_{i}}\right\|
$$

Therefore,

$$
\begin{gathered}
\left\|x_{k_{i}}-x_{k_{j}-m}\right\| \leq\left\|x_{k_{i}}-R_{i} \bar{y}\right\|+\left\|R_{i} \bar{y}-x_{k_{j}-m}\right\| \\
\leq 2^{-i}\left\|x_{k_{i}}\right\|+\left\|x_{k_{j}}\right\|-\frac{3}{4}\left\|x_{k_{i}}\right\|
\end{gathered}
$$


by eqns. 2.5) and (2.7). Thus, for $i \geq 1$ and $j$ large enough,

$$
\left\|x_{k_{i}}-x_{k_{j}-m}\right\| \leq\left\|x_{k_{j}}\right\|-\frac{1}{4}\left\|x_{k_{i}}\right\|,
$$

which proves eq. (2.4).

\section{Proof of the main theorem}

Given a function $g: X \rightarrow \mathbb{R}$, we define the epigraph of $g$ to be

$$
\text { epi } g=\left\{(x, t) \mid x \in \mathbb{R}^{n}, t \in \mathbb{R}, t \geq g(x)\right\} .
$$

We say that $g$ is convex if epi $g$ is a convex subset of $X \times \mathbb{R}$. Note that any horofunction $h \in X(\infty)$ is convex. After all, suppose that $\left(y_{1}, t_{1}\right),\left(y_{2}, t_{2}\right) \in$ epi $h$. Then, $t_{1} \geq h\left(y_{1}\right)$ and $t_{2} \geq h\left(y_{2}\right)$. Suppose that $0<\lambda<1$. By eq. (2.1),

$$
h\left(\lambda y_{1}+(1-\lambda) y_{2}\right)=\lim _{k \rightarrow \infty}\left\|\lambda y_{1}+(1-\lambda) y_{2}-x_{k}\right\|-\left\|x_{k}\right\|
$$

for some sequence $\left\langle x_{k}\right\rangle_{k \geq 0}$ in $X$. Note that

$$
\left\|\lambda y_{1}+(1-\lambda) y_{2}-x_{k}\right\| \leq \lambda\left\|y_{1}-x_{k}\right\|+(1-\lambda)\left\|y_{2}-x_{k}\right\|
$$

and therefore,

$$
\begin{gathered}
h\left(\lambda y_{1}+(1-\lambda) y_{2}\right) \leq \lim _{k \rightarrow \infty} \lambda\left(\left\|y_{1}-x_{k}\right\|-\left\|x_{k}\right\|\right)+(1-\lambda)\left(\left\|y_{2}-x_{k}\right\|-\left\|x_{k}\right\|\right) \\
=\lambda h\left(y_{1}\right)+(1-\lambda) h\left(y_{2}\right) .
\end{gathered}
$$

From this it follows that $\lambda t_{1}+(1-\lambda) t_{2} \geq h\left(\lambda y_{1}+(1-\lambda) y_{2}\right)$ and therefore

$$
\left(\lambda y_{1}+(1-\lambda) y_{2}, \lambda t_{1}+(1-\lambda) t_{2}\right) \in \text { epi } h .
$$

The following lemma is an immediate consequence of Corollary 12.1.2 in [17, although we give a proof here for the sake of completeness.

Lemma 3.1. For any horofunction $h \in X(\infty)$ there exist some $\varphi \in X^{*}$ and $b \in \mathbb{R}$ such that $h(x) \geq-\varphi(x)+b$ for every $x \in X$.

Proof. Since $h$ is a continuous convex function, epi $h$ is a closed convex set. Fix an $x_{0} \in X$ and $t_{0} \in \mathbb{R}$ such that $t_{0}<h\left(x_{0}\right)$ and therefore $\left(x_{0}, t_{0}\right) \notin$ epi $h$. Since epi $h$ is convex, the Hahn-Banach theorem implies that there is a linear functional $\Psi$ on $X \times \mathbb{R}$ and a constant $c \in \mathbb{R}$ such that $\Psi((x, t)) \geq c$ for all $(x, t) \in$ epi $h$ and $\Psi\left(\left(x_{0}, t_{0}\right)\right)<c$. Note that the linear functional $\Psi$ can be written $\Psi((x, t))=$ $\psi(x)+a t$, where $\psi \in X^{*}$ and $a \in \mathbb{R}$. In particular, $\psi(x)+a h(x) \geq c$ for all $x \in X$. Since $\psi\left(x_{0}\right)+a h\left(x_{0}\right) \geq c$ while $\psi\left(x_{0}\right)+a t_{0}<c$, it follows that $a\left(h\left(x_{0}\right)-t_{0}\right)>0$. Thus, $a>0$. By rearranging terms, we can see that $h(x) \geq a^{-1} c-a^{-1} \psi(x)$. To complete the proof, we let $\varphi=a^{-1} \psi$ and $b=a^{-1} c$.

Remark 3.1. The conclusion of Lemma 3.1 holds for any continuous convex function. Readers familiar with convex functions may note that we could have proven Lemma 3.1 by using the fact that every continuous convex function is subdifferentiable (Theorem 23.4, [17]).

We are now ready to prove Theorem 1.2 , 
Proof of Theorem [1.2, By Theorem 2.1 there is a horofunction $h \in X(\infty)$ such that

$$
\lim _{k \rightarrow \infty} h\left(f^{k}(x)\right)=-\infty, \text { for all } x \in C .
$$

Lemma 3.1 implies that there is some $\varphi \in X^{*}$ and $b \in \mathbb{R}$ such that $h(x) \geq-\varphi(x)+b$ for all $x \in X$. Thus, $\varphi(x) \geq-h(x)+b$ for all $x \in X$. It immediately follows that $\lim _{k \rightarrow \infty} \varphi\left(f^{k}(x)\right)=\infty$ for all $x \in C$.

\section{ACKNOWLEDGEMENT}

The author would like to thank the reviewer for several helpful comments and suggestions.

\section{REFERENCES}

1. A. F. Beardon. Iteration of contractions and analytic maps, J. London Math. Soc. 41 (1990), 141-150. MR1063551(91i:51025)

2. D. Burago, Y. Burago, and S. Ivanov. A Course in Metric Geometry, Graduate Studies in Mathematics, vol. 33, Amer. Math. Soc., Providence, RI, 2001. MR1835418 (2002e:53053)

3. A. Całka. On conditions under which isometries have bounded orbits, Colloq. Math. 48 (1984), 219-227. MR758530 (85m:54027)

4. C. M. Dafermos and M. Slemrod. Asymptotic behavior of nonlinear contraction semigroups, J. Functional Anal. 13 (1973), 97-106. MR0346611(49:11336)

5. M. Edelstein. On non-expansive mappings of Banach spaces, Proc. Camb. Phil. Soc. 60 (1964), 439-447. MR0164222 (29:1521)

6. J. Gunawardena and M. Keane. On the existence of cycle times for some nonexpansive maps, Technical Report HPL-BRIMS-95-003, Hewlett-Packard Labs, 1995.

7. A. Karlsson. Non-expanding maps and Busemann functions, Ergodic Theory and Dynamical Systems 21 (2001), 1447-1457. MR.1855841(2002f:37055)

8. A. Karlsson, V. Metz and G. Noskov. Horoballs in simplices and Minkowski spaces, Int. J. Math. Math. Sci. (2006), Art. ID 23656, 20 pages. MR2268510 (2007k:53047)

9. E. Kohlberg. Invariant half-lines of nonexpansive piecewise-linear transformations, Math. Oper. Res. 5 (1980), 366-372. MR.594851 (82a:55005)

10. E. Kohlberg and A. Neyman. Asymptotic behavior of nonexpansive mappings in normed linear spaces, Israel J. Math. 38 (1981), 269-275. MR617673 (83g:47056)

11. B. Lins. A Denjoy-Wolff theorem for Hilbert metric nonexpansive maps on polyhedral domains, Math. Proc. Camb. Phil. Soc. 143 (2007), 157-164. MR2340981(2008i:47102)

12. R. D. Nussbaum. Fixed point theorems and Denjoy-Wolff theorems for Hilbert's projective metric in infinite dimensions, Topological Methods in Nonlinear Analysis 29 (2007), 199-249. MR.2345061

13. A. T. Plant and S. Reich. The asymptotics of nonexpansive iterations, J. Functional Anal. 54 (1983), 308-319. MR724526 (85a:47055)

14. S. Reich. Fixed point iterations of nonexpansive mappings, Pacific J. Math. 60 (1975), 195198. MR0428130 (55:1159)

15. S. Reich. Extension problems for accretive sets in Banach spaces, J. Functional Anal. 26 (1977) 378-395. MR0477893 (57:17393)

16. S. Reich. On the asymptotic behavior of nonlinear semigroups and the range of accretive operators, J. Math. Anal. Appl. 79 (1981), 113-126. MR603380 (82c:47066)

17. R. T. Rockafeller. Convex Analysis, Princeton University Press, Princeton, NJ, 1970. MR0274683 (43:445)

18. B. D. Rouhani and W. A. Kirk. Asymptotic properties of nonexpansive iterations in reflexive spaces, J. Math. Anal. Appl. 236 (1999), 281-289. MR.1704583 (2001a:47056)

19. C. Walsh. The horofunction boundary of finite-dimensional normed spaces, Math. Proc. Camb. Phil. Soc. 142 (2007), 497-507. MR2329698(2008e:53150)

Department of Mathematics and Computer Science, Hampden-Sydney College, HAMPDEN-SYDNEY, VIRGINIA 23943

E-mail address: blins@hsc.edu 